\title{
Factors Associated with Good Patient Outcomes Following Convalescent Plasma in COVID-19: A Prospective Phase II Clinical Trial
}

\author{
Danyal Ibrahim (D) - Latha Dulipsingh · Lisa Zapatka • \\ Reginald Eadie $\cdot$ Rebecca Crowell $\cdot$ Kendra Williams · Dorothy B. Wakefield • \\ Lisa Cook · Jennifer Puff · Syed A. Hussain
}

Received: August 3, 2020 / Accepted: September 10, 2020 / Published online: September 20, 2020

(C) The Author(s) 2020

\begin{abstract}
Introduction: Coronavirus disease 2019 (COVID-19) is a viral respiratory syndrome caused by severe acute respiratory syndrome coronavirus 2 (SARS-CoV-2). This novel virus was discovered in Wuhan City, Hubei Province, China, in December 2019. As of September 6, 2020 , confirmed cases have risen to more than $27,000,000$ worldwide and more than 885,000 people have died. Currently, no cure or standard treatment for COVID-19 exists. We conducted a prospective single-arm open-label phase II clinical trial assessing the safety and efficacy of convalescent plasma in hospitalized patients with COVID-19.

Methods: Convalescent plasma with sufficient total anti-SARS-CoV-2 IgG titer (1:320) obtained from recovered donors was administered to
\end{abstract}

Digital Features To view digital features for this article go to https://doi.org/10.6084/m9.figshare.12933338.

D. Ibrahim $(\bowtie) \cdot$ L. Zapatka $\cdot$ R. Eadie

S. A. Hussain

Trinity Health of New England, Hartford, CT, USA

e-mail: dibrahim@trinityhealthofne.org

L. Dulipsingh · L. Cook · J. Puff

Diabetes Center, Saint Francis Hospital, Hartford, CT, USA

R. Crowell · K. Williams - D. B. Wakefield Research Department, Saint Francis Hospital, Hartford, CT, USA adult patients with either severe or critical COVID-19 illness. Primary outcomes were adverse events in association with plasma administration, and hospital mortality. Secondary outcomes included disease progression, recovery, length of stay, and hospital discharge. Results: Of the 38 patients included in the analysis, 24 (63\%) recovered and were discharged, and 14 (37\%) died. Patients who received convalescent plasma early in the disease course (severe illness group) as compared to the patients that received convalescent plasma later in the disease progression (critical illness group) had significantly lower hospital mortality $13 \%$ vs $55 \%(p<0.02)$ and shorter mean hospital length of stay 15.4 vs 33 days $(p<0.01)$. One patient experienced a transient transfusion reaction. No other adverse effects of convalescent plasma infusion were observed.

Conclusions: Our results suggest that convalescent plasma with adequate anti-SARS-CoV-2 antibody titer is safe and has the potential for positive impact on clinical outcomes including recovery and survival if given to patients early in the course of COVID-19 disease.

Trial Registration: ClinicalTrials.gov. Identifier, NCT04343261, IND \#19805.

Keywords: Adult respiratory distress syndrome; COVID-19; COVID-19 serotherapy; Hospital mortality; Length of stay; Phase II clinical trial; Plasma; Prospective studies; Severe acute respiratory syndrome coronavirus 2; Ventilation 


\section{Key Summary Points}

Coronavirus disease 2019 (COVID-19) is a viral respiratory syndrome caused by severe acute respiratory syndrome coronavirus 2 (SARS-CoV-2).

This novel virus was discovered in Wuhan City, Hubei Province, China, in December 2019, and as of September 6, 2020, confirmed cases have risen to more than $27,000,000$ worldwide and more than 885,000 people have died.

Currently, no cure or standard treatment for COVID-19 exists.

We conducted a prospective single-arm open label phase II clinical trial assessing the safety and efficacy of convalescent plasma in hospitalized patients with COVID-19.

Patients who received convalescent plasma with adequate amount of antiSARS-CoV-2 antibodies early in the disease course (severe illness group) as compared to the patients that received convalescent plasma later in disease progression (critical illness group) had significantly lower hospital mortality $13 \%$ vs $55 \%$ $(p<0.02)$ and shorter mean hospital length of stay 15.4 vs 33 days $(p<0.01)$.

Of the 38 patients that received convalescent plasma, only one patient experienced a transient transfusion reaction.

\section{DIGITAL FEATURES}

This article is published with digital features to facilitate understanding of the article. To view digital features for this article go to https://doi. org/10.6084/m9.figshare.12933338.

\section{INTRODUCTION}

Coronavirus disease 2019 (COVID-19) is a viral respiratory syndrome caused by severe acute respiratory syndrome coronavirus 2 (SARS-CoV2). This novel virus was discovered in Wuhan City, Hubei Province, China, in December 2019. As of September 6, 2020, confirmed cases have risen to more than $27,000,000$ worldwide and more than 885,000 people have died [1]. Currently, no cure or standard treatment for COVID-19 exists.

The majority of people with COVID-19 experience an asymptomatic, mild, or manageable course of disease $[2,3]$. The most common symptoms include fever, cough, fatigue, dyspnea, headache, diarrhea, myalgia, and/or loss of taste and smell [4, 5]. However, $19 \%$ of those who are infected with the virus become severely or critically ill [2]. Life-threatening illness occurs when the virus triggers a progressive hyper-immune response or "cytokine storm" progressing to acute respiratory distress syndrome (ARDS), cardiac injury, thrombotic complications, septic shock, and/or organ failure [6-9]. Estimated mortality among patients admitted to the intensive care unit (ICU) with severe or critical illness ranges from $34.8 \%$ to $41.6 \%[10,11]$. Higher mortality rates $(48-55 \%)$ were observed in patients with ARDS, needing invasive ventilatory support $[10,11]$. Risk of death and disease severity increase with older age, obesity, and chronic disease such as hypertension, diabetes, and cardiovascular disease [7-9, 12-14].

In March of 2020, the US Food and Drug Administration (FDA) solicited investigational new drug applications to test the safety and efficacy of convalescent plasma therapy for patients with severe or life-threatening COVID19 [15]. Convalescent plasma is derived from the blood of recovered patients and is a rich source of antibodies. When administered to patients who are ill with the same disease, the plasma may aid recovery by conferring passive immunity and neutralizing the pathogen [15]. The therapy showed promise during outbreaks of other novel viral respiratory syndromes, including two caused specifically by 
coronavirus [SARS-CoV in 2003 and Middle East respiratory syndrome (MERS) in 2012] [16, 17]. Data showed that convalescent plasma might be most effective when given earlier in the course of disease, but research was limited to small observational studies and much remains unknown [16, 17].

Preliminary data from clinical trials and observational studies targeting COVID-19 suggest that administration of convalescent plasma may reduce mortality, hospital length of stay, and time on mechanical ventilation with minimal adverse side effects in patients with severe or life-threatening disease [18-23]. Consistent with earlier studies, treatment may be most efficacious for severe COVID-19 when administered closer to symptom onset [21-25]. The purpose of this study is to describe the course of illness among 38 patients hospitalized with severe or life-threatening COVID-19 who received convalescent plasma as part of an FDAapproved phase 2 clinical trial. Specifically, the study will assess their hospital course in the context of demographics, disease onset, symptomology, illness severity, and disease progression.

\section{METHODS}

This study is an FDA-approved prospective single-arm open-label phase II clinical trial (NCT04343261) assessing the safety and efficacy of convalescent plasma (IND \#19805) on the clinical course of adult patients hospitalized with severe COVID-19. The study protocol was approved by the Trinity Health of New England Institutional Review Board (\#SFH-20-23). The research study was performed in accordance with the Helsinki Declaration of 1964, and its later amendments. Informed consent was provided by either the patient or the patient's legally authorized representative (LAR).

\section{Patients}

Subjects were recruited from four regional hospitals in Connecticut and Massachusetts between April 20, 2020 and June 8, 2020. Patients were considered eligible for the study if they were between the ages of 18 and 90, hospitalized, severely or critically ill with confirmed COVID-19 through nasopharyngeal swab real-time PCR (RT-PCR). Illness severity was defined as follows: mild COVID-19 was defined as symptoms with no clinical signs of moderate, severe, or critical disease; moderate illness was defined as respiratory rate $\geq 20$ breaths per minute and oxygen saturation > 93\%; severe illness was defined as any of the following: respiratory frequency $\geq 30 / \mathrm{min}$, blood oxygen saturation $\leq 93 \%$, partial pressure of arterial oxygen to fraction of inspired oxygen ratio $<300$, and lung infiltrates $>50 \%$ within 24-48 h; evidence of critical illness included respiratory failure, septic shock, or multi-organ dysfunction or failure $[15,26]$.

Subjects who met eligibility criteria were referred by their treating physicians. Patients were enrolled regardless of previous treatment or therapies for COVID-19, including experimental medications and therapies administered off-label. Informed consent was provided by either the patient or the patient's LAR. Once a patient or the patient's LAR provided informed consent and the patient's ABO blood type was determined, compatible convalescent plasma was administered in two consecutive $200-\mathrm{mL}$ infusions. Each unit was transfused for the duration of $1 \mathrm{~h}, 1-2 \mathrm{~h}$ apart. If the patient received plasma with undetectable total antiSARS-CoV-2 antibodies, the patient was redosed with a unit of plasma with adequate antibody titer (1:320). Recipients were monitored and all adverse reactions or events were recorded whether or not they were related to the plasma infusion. The protocol was approved by the Trinity Health of New England Institutional Review Board (\#SFH-20-23).

\section{Convalescent Plasma}

Convalescent plasma was obtained from adult donors who were confirmed positive and had recovered from SARS-CoV-2. All donors screened negative for the virus using a nasal swab (RT-PCR) and had total anti-SARS-CoV-2 titer $>6.5$ arbitrary units per milliliter $(\mathrm{AU} / \mathrm{mL}$; equivalent to $1: 320)$. Plasma was collected by 
apheresis at an established blood donation center following standard operating procedures and 21 CFR 630.10 requirements. Plasma was frozen within $24 \mathrm{~h}$ of collection and labeled for investigational use and $\mathrm{ABO}$ typing.

\section{Data and Data Sources}

Demographic, clinical, and outcomes data were prospectively collected from electronic patient medical records at each of the four hospitals. Descriptive data included sex, age, race, ethnicity, smoking status, functional status, comorbidities, living situation, and means of arrival to the hospital. Initial presentation to the emergency department included self-reported symptoms, vital signs, degree of respiratory distress, and need for oxygen supplementation and resuscitation. Initial chest $\mathrm{X}$-ray findings, and laboratory markers of sepsis, inflammatory response, immune deficiency, and organ dysfunction were recorded. The clinical course during hospital stay was prospectively captured by tracking changes in oxygenation $\left(\mathrm{FiO}_{2}\right)$, need for invasive ventilation, ICU-level care, and types of essential medications given. Patient clinical status progression and recovery were prospectively monitored by capturing days on invasive ventilation, intubation, extubation, discharge alive, and death during hospitalization.

\section{Outcomes and Data Analysis}

Primary clinical outcomes were rate of adverse events associated with convalescent plasma administration, and hospital mortality. Secondary outcomes included disease progression, recovery, length of hospital stay, and hospital discharge. Primary and secondary clinical outcomes were compared between two groups based on severity of illness $[15,26]$ at the time of plasma infusion: (1) patients with severe illness, who had not progressed to ARDS at the time of enrollment, and (2) patients whose condition had progressed to critical illness at the time of enrollment. Patients were excluded from the analysis if they were transferred to another acute hospital; did not receive convalescent plasma with adequate antibody titer; or care was withdrawn and patient received comfort care only within 5 days of plasma administration.

\section{Statistical Analysis}

Descriptive statistics included means, medians, and proportions as appropriate based on variable, sample size, and distribution. Descriptive variables included demographic characteristics, clinical parameters, and time from illness onset and hospitalization to plasma transfusion. As a result of the small sample size, both parametric and nonparametric statistics were used in the analysis as appropriate. Continuous variables were compared using $t$ tests, and categorical variables using chi-square analyses and Fisher's exact test when cell sizes were small. SAS software version 9.4 (SAS Institute Inc., Cary, NC, USA) was used for analyses. Outcomes were considered statistically significant at $p<0.05$.

\section{RESULTS}

\section{Plasma Recipients}

A total of 46 patients (Fig. 1) with RT-PCR-confirmed COVID-19 were enrolled in the study. Eight of $46(17 \%)$ patients were excluded from

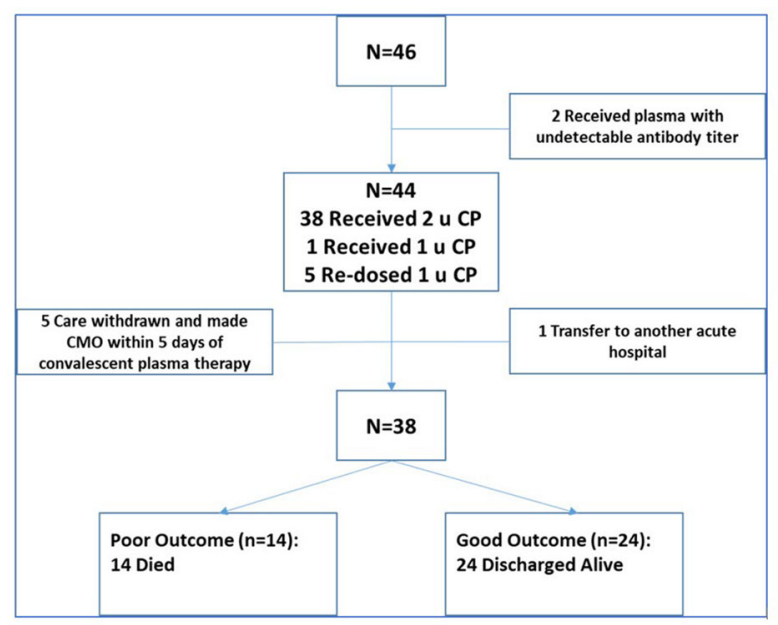

Fig. 1 Flowchart of patients and outcomes. CP convalescent plasma, CMO comfort care only 
the analysis for the following reasons: two received convalescent plasma with no detectable antibody titer; one was transferred to another hospital; five were made comfort care only (CMO) and medical care was withdrawn within 5 days of plasma administration. The remaining patients $(n=38)$ included in this analysis received convalescent plasma with adequate total anti-SARS-CoV-2 antibody titer of $1: 320$ (32 received 2 units, 5 were re-dosed with 1 unit, 1 received 1 unit).

Patient demographics, clinical presentation, hospital course, and clinical outcomes are shown in Tables $1,2,3,4$, and 5. Mean age was 63 years (95\% CI 59-70), 53\% were male, 34\% black, $32 \%$ white, and $34 \%$ were Hispanic; $61 \%$ of the patients were from Connecticut, $37 \%$ from Harford County, 21\% New Haven County, and $40 \%$ were from Hampden County, Massachusetts (Table 1). More than $68 \%$ had been diagnosed with hypertension and nearly half (47.4\%) with diabetes mellitus; overall, 31.5\% had three or more comorbidities (Table 2). As shown in Table 3, mean days from onset of symptoms to hospitalization was 7.3 days (95\% CI 6.4-8.2), with the most common symptoms at admission being fever, cough, and dyspnea. With the exception of one patient who arrived in critical condition, subjects presented initially to the hospital with moderate to severe COVID19 pneumonia without evidence of ARDS or requiring invasive ventilation support at the time of admission (Table 4). The most common laboratory abnormalities on admission included severe rise in inflammatory markers [C-reactive protein $(\mathrm{CRP}) \geq 10 \mathrm{mg} / \mathrm{dL}](66 \%)$, lymphopenia (absolute lymphocyte count $<1000$ per microliter) $(50 \%)$, and hyponatremia $(\mathrm{Na}<135 \mathrm{mEq} /$ L) (47\%)—see Table 4.

\section{Severe and Critical Illness Groups}

At the time of plasma infusion, 16 patients (42\%) met criteria for severe illness. These

Table 1 Demographic characteristics of convalescent plasma recipients

\begin{tabular}{lllll}
\hline & Overall, $\boldsymbol{n}=\mathbf{3 8}$ & Severe, 16 (42) & Critical, 22 (58) & $\boldsymbol{p}$ value \\
\hline Age, mean (SD) & $63(12)$ & $65(11)$ & $61(13)$ & 0.30 \\
Age less than 70 & $28(74)$ & $11(69)$ & $17(77)$ & 0.56 \\
Gender (female) & $18(47)$ & $8(50)$ & $10(46)$ & 0.78 \\
Race & & & & 0.92 \\
Black & $13(34)$ & $6(38)$ & $7(32)$ & \\
White & $12(32)$ & $5(31)$ & $7(32)$ & $8(36)$ \\
Other & $13(34)$ & $5(31)$ & $9(41)$ & 0.31 \\
Ethnicity (Hispanic) & $13(34)$ & $4(25)$ & & 0.45 \\
County & $15(40)$ & & $8(36)$ & \\
Hampden County & $14(37)$ & $7(44)$ & $7(32)$ & \\
Hartford County & $8(21)$ & $2(44)$ & $6(27)$ & \\
New Haven County & $1(3)$ & $0(0)$ & $4(5)$ & \\
Tolland County & $8(21)$ & $4(25)$ & $7(44)$ & $10(45)$ \\
Insurance-Medicaid or self-pay & $17(45)$ & & \\
Marital status-married & &
\end{tabular}

Values are no. (\%) except as indicated 
Table 2 Comorbidities and home medications of convalescent plasma recipients

\begin{tabular}{|c|c|c|c|c|}
\hline & Overall, $n=38$ & Severe, $16(42)$ & Critical, 22 (58) & $p$ value \\
\hline BMI, mean $(S D)$ & $33.6(6.5)$ & $34.4(7.9)$ & $33(5.4)$ & 0.53 \\
\hline Smoking status (smoker) & $4(11)$ & $2(13)$ & $2(9)$ & 0.87 \\
\hline ABO blood type-A group & $9(24)$ & $5(31)$ & $4(18)$ & 0.48 \\
\hline \multicolumn{5}{|l|}{ Comorbidities } \\
\hline COPD/asthma & $15(39)$ & $7(44)$ & $8(36)$ & 0.64 \\
\hline Obstructive sleep apnea & $5(13)$ & $1(6)$ & $4(18)$ & 0.37 \\
\hline Hypertension & $26(68)$ & $9(56)$ & $17(77)$ & 0.17 \\
\hline Diabetes mellitus & $18(47)$ & $8(50)$ & $10(45)$ & 0.78 \\
\hline Coronary artery disease & $5(13)$ & $1(6)$ & $4(18)$ & 0.37 \\
\hline Chronic kidney disease & $5(13)$ & $2(13)$ & $3(14)$ & 1.00 \\
\hline Cancer & $4(11)$ & $2(13)$ & $2(9)$ & 1.00 \\
\hline VTE & $3(8)$ & $2(13)$ & $1(5)$ & 0.56 \\
\hline Multiple comorbidities & & & & 0.46 \\
\hline $0-2$ & $26(68)$ & $12(75)$ & $14(64)$ & \\
\hline $3-7$ & $12(32)$ & $4(25)$ & $8(36)$ & \\
\hline \multicolumn{5}{|l|}{ Home medications } \\
\hline $\mathrm{ARB}$ & $11(29)$ & $6(38)$ & $5(23)$ & 0.32 \\
\hline ACEi & $8(21)$ & $3(19)$ & $5(23)$ & 1.00 \\
\hline CCB & $9(24)$ & $2(13)$ & $7(32)$ & 0.25 \\
\hline NSAIDs & $14(37)$ & $6(38)$ & $8(36)$ & 0.94 \\
\hline Steroids & $4(11)$ & $1(6)$ & $3(14)$ & 0.63 \\
\hline
\end{tabular}

Values are no. (\%) except as indicated

$A R B$ angiotensin II receptor blocker, $A C E i$ angiotensin converting enzyme inhibitor, $C C B$ calcium channel blocker, NSAIDs non-steroidal anti-inflammatory drugs, VTE venous thromboembolism

patients were enrolled in the study and received convalescent plasma earlier in their hospital and disease course on average 4.6 days (95\% CI 2.9-6.3) following hospital admission, and 12.6 days (95\% CI 10-15.2) following symptoms' onset while on high-flow oxygen supplementation prior to any evidence of ARDS. The remaining 22 patients (58\%) met the criteria for critical illness at the time of convalescent plasma therapy. They enrolled in the study and received convalescent plasma later in their hospital and disease course on average 16.4 days (95\% CI 13-19.8) following hospital admission, and 23.1 days (95\% CI 19.5-26.7) following symptoms' onset after developing ARDS and had been on ventilation support for an average of 10.6 days (95\% CI 7.3-13.9).

The two cohorts were comparable in demographics; comorbidities and home medications; pre-illness functional status; onset of symptoms to seeking hospital care; initial clinical presentation and findings; initial disease severity; and 
Table 3 Characteristics and symptoms at presentation to the hospital among convalescent plasma recipients

\begin{tabular}{|c|c|c|c|c|}
\hline & Overall, $n=38$ & Severe, $16(42)$ & Critical, 22 (58) & $p$ value \\
\hline Mode of arrival-EMS & $28(74)$ & $11(69)$ & $17(77)$ & 0.56 \\
\hline Patient origin & & & & 0.68 \\
\hline Home & $32(84)$ & $13(81)$ & $19(86)$ & \\
\hline Skilled nursing facility & $6(16)$ & $3(19)$ & $3(14)$ & \\
\hline Functional status-independent of ADLs & $33(87)$ & $13(81)$ & $20(91)$ & 0.63 \\
\hline Source of exposure to COVID-19 & & & & 0.30 \\
\hline Family & $10(26)$ & $3(19)$ & $7(32)$ & \\
\hline Long-term care facility & $5(13)$ & $3(19)$ & $2(9)$ & \\
\hline Work & $6(16)$ & $1(6)$ & $5(23)$ & \\
\hline No known source & $17(45)$ & $9(56)$ & $8(36)$ & \\
\hline Onset of symptoms (days), mean (SD) & $7.3(2.8)$ & $8(3.1)$ & $6.7(2.6)$ & 0.17 \\
\hline \multicolumn{5}{|l|}{ Symptoms reported on presentation to hospital } \\
\hline Fever (temperature $\geq 38^{\circ} \mathrm{C}$ or subjective) & $30(79)$ & $14(88)$ & $16(73)$ & 0.43 \\
\hline Cough & $35(92)$ & $15(94)$ & $20(91)$ & 1.00 \\
\hline Dyspnea & $37(97)$ & $15(94)$ & $22(100)$ & 0.42 \\
\hline Headache & $9(24)$ & $5(31)$ & $4(18)$ & 0.45 \\
\hline Loss of appetite & $17(45)$ & $6(38)$ & $11(50)$ & 0.52 \\
\hline Fatigue & $24(63)$ & $11(69)$ & $13(59)$ & 0.74 \\
\hline Body aches & $13(34)$ & $5(31)$ & $8(36)$ & 1.00 \\
\hline Chest pain & $9(24)$ & $4(25)$ & $5(23)$ & 0.87 \\
\hline GI symptoms & $17(45)$ & $7(44)$ & $10(45)$ & 0.92 \\
\hline Loss of taste or smell & $3(8)$ & $2(13)$ & $1(5)$ & 0.56 \\
\hline Confusion & $6(16)$ & $4(25)$ & $2(9)$ & 0.22 \\
\hline
\end{tabular}

Values are no. (\%) except as indicated

$E M S$ emergency medical services, $A D L s$ activities of daily living

the care they received during their hospitalization including essential medications (see Tables 1, 2, 3, 4 and 5). Clinically, hyponatremia on initial hospital presentation was more prevalent in the severe illness group $(p=0.047)$. Vasopressors $(p<0.01)$, hydroxychloroquine $(p<0.01)$, and antibiotics $(p<0.01)$ were more frequently used during hospitalization in the critical illness group. Renal replacement therapy was utilized at higher rate in the critical illness group but did not reach statistical significance $(p=0.05)$.

\section{Primary Outcomes}

One patient in the severe illness group experienced a transient transfusion reaction (fever and hematuria) within $2 \mathrm{~h}$ of plasma infusion. No other adverse effects of convalescent plasma infusion were observed. Of the 38 patients 
Table 4 Clinical presentation at admission of convalescent plasma recipients

\begin{tabular}{|c|c|c|c|c|}
\hline & Overall, $n=38$ & Severe, $16(42)$ & Critical, $22(58)$ & $p$ value \\
\hline Disease severity (FDA classification) on admission & & & & 0.14 \\
\hline Moderate & 5 & $4(25)$ & $1(5)$ & \\
\hline Severe & 32 & $12(75)$ & $20(90)$ & \\
\hline Critical & 1 & $0(0)$ & $1(5)$ & \\
\hline RA O2Sat $\leq 85 \%$ & $18(47)$ & $6(38)$ & $12(55)$ & 0.30 \\
\hline Oxygen support on admission & & & & 0.61 \\
\hline Low flow nasal & $27(71)$ & $11(69)$ & $16(72)$ & \\
\hline High flow nasal & $10(26)$ & $5(31)$ & $5(23)$ & \\
\hline Invasive ventilation & $1(3)$ & $0(0)$ & $1(5)$ & \\
\hline Chest X-ray with typical COVID-19 findings ${ }^{a}$ & $35(92)$ & $14(88)$ & $21(95)$ & 0.56 \\
\hline Febrile (temperature $\geq 38^{\circ} \mathrm{C}$ ) & $10(26)$ & $6(38)$ & $4(18)$ & 0.27 \\
\hline Hypotension $^{\mathrm{b}}$ & $1(3)$ & $0(0)$ & $1(5)$ & 1.00 \\
\hline Lymphopenia $^{c}$ & $19(50)$ & $9(56)$ & $10(45)$ & 0.51 \\
\hline Rise in sepsis markers ${ }^{\mathrm{d}}$ & $13(34)$ & $4(25)$ & $9(41)$ & 0.31 \\
\hline Severe rise in inflammatory markers ${ }^{\mathrm{e}}$ & $25(66)$ & $10(63)$ & $15(68)$ & 0.71 \\
\hline Transaminitis $^{\mathrm{f}}$ & $9(24)$ & $4(25)$ & $5(23)$ & 1.00 \\
\hline $\mathrm{AKI}^{\mathrm{g}}$ & $10(26)$ & $7(44)$ & $3(14)$ & 0.06 \\
\hline Hyponatremia $^{\mathrm{h}}$ & $18(47)$ & $11(69)$ & $7(32)$ & 0.047 \\
\hline Hypokalemia $^{\mathrm{i}}$ & $7(18)$ & $1(6)$ & $6(27)$ & 0.20 \\
\hline Troponin leak $^{\mathrm{i}}$ & $7(18)$ & $2(13)$ & $5(23)$ & 0.68 \\
\hline
\end{tabular}

Values are no. (\%) except as indicated. Bold text $=$ statistically significant $R A$ O2Sat room-air oxygen saturation, $A K I$ acute kidney injury

${ }^{a}$ Multifocal peripheral consolidation and/or multifocal rounded opacities and nodules

${ }^{\text {b }}$ Mean arterial blood pressure $<60 \mathrm{mmHg}$

c Absolute lymphocyte count $<1000$ per microliter

d Serum lactate $>2.2 \mathrm{mmol} / \mathrm{L}$

e C-reactive protein $\geq 10 \mathrm{mg} / \mathrm{dL}$

$\mathrm{f} \geq 5 \times$ rise in serum ALT level

g eGFR $<60 \mathrm{~mL} / \mathrm{min} / 1.73 \mathrm{~m}^{2}$

h Serum sodium $<135 \mathrm{mEq} / \mathrm{L}$

i Serum potassium $<3.5 \mathrm{mEq} / \mathrm{L}$

i Serum troponin $>0.04 \mathrm{ng} / \mathrm{mL}$

included in the analysis, 24 (63\%) recovered and were discharged from the hospital, and 14 (37\%) died. Patients who died included two in the severe illness group and 12 in the critical illness group. The difference in mortality (13\% severe vs $55 \%$ critical) was statistically significant $(p=0.02)$. Overall, patients who survived $(n=24)$ regardless of disease severity at time of infusion received convalescent plasma earlier in their course of disease (mean 15.3 days, SD 6.9) 
Table 5 Patient outcomes and hospital course

\begin{tabular}{|c|c|c|c|c|}
\hline & Overall, $n=38$ & Severe $16(42)$ & Critical, $22(58)$ & $p$ value \\
\hline \multicolumn{5}{|l|}{ Outcome } \\
\hline Mortality & $14(37)$ & $2(13)$ & $12(55)$ & 0.02 \\
\hline Length of stay (days), mean (SD) & $26(15)$ & $15.4(11.6)$ & $33(12.9)$ & $<0.01$ \\
\hline Symptom onset to CP (days), mean (SD) & $18.7(9.0)$ & $12.6(5.3)$ & $23.1(8.6)$ & $<0.01$ \\
\hline Symptom onset to $\mathrm{CP}$ admin $\leq 15$ days & $17(45)$ & $13(81)$ & $4(18)$ & $<0.01$ \\
\hline Hospital days prior to CP admin, mean (SD) & $11.4(8.8)$ & $4.6(3.4)$ & $16.4(8.1)$ & $<0.01$ \\
\hline Hospital days after CP admin, mean (SD) & $14.2(11.5)$ & $10.9(10.5)$ & $16.5(11.9)$ & $<0.01$ \\
\hline ARDS prior to $\mathrm{CP}$ admin & $22(58)$ & $0(0)$ & $22(100)$ & $<0.01$ \\
\hline ARDS during hospitalization & $26(68)$ & $4(25)$ & $22(100)$ & $<0.01$ \\
\hline Invasive mechanical ventilation & $25(66)$ & $3(19)$ & $22(100)$ & $<0.01$ \\
\hline Ventilator days, mean (SD) & $20.3(10.3)$ & $16(12.1)$ & $21(10.2)$ & 0.45 \\
\hline \multicolumn{5}{|l|}{ Other interventions and medications } \\
\hline Renal replacement therapy & $9(24)$ & $1(6)$ & $8(36)$ & 0.05 \\
\hline Antibiotics & $32(84)$ & $10(63)$ & $22(100)$ & $<0.01$ \\
\hline Antifungals & $4(11)$ & $0(0)$ & $4(18)$ & 0.12 \\
\hline Azithromycin & $16(42)$ & $4(25)$ & $12(55)$ & 0.07 \\
\hline Hydroxychloroquine & $17(45)$ & $3(19)$ & $14(64)$ & $<0.01$ \\
\hline IL-6 Inhibitors & $10(26)$ & $3(19)$ & $7(32)$ & 0.47 \\
\hline Remdesivir & $4(11)$ & $3(19)$ & $1(5)$ & 0.29 \\
\hline Vasopressors & $20(53)$ & $3(19)$ & $17(77)$ & $<0.01$ \\
\hline Steroids & $22(58)$ & $7(44)$ & $15(68)$ & 0.13 \\
\hline Anticoagulants & $31(82)$ & $13(81)$ & $18(82)$ & 1.00 \\
\hline Zinc & $17(45)$ & $6(38)$ & $11(50)$ & 0.44 \\
\hline
\end{tabular}

Values are no. (\%) except as indicated

ARDS acute respiratory distress syndrome, $C P$ convalescent plasma

Bold text $=$ statistically significant

and hospital stay (8.4 days, SD 6.8) compared to those who died $(n=14)$ with mean durations of (24.5 days, SD 9.6), (16.6 days, SD 9.5) respectively.

\section{Secondary Outcomes}

Among patients with severe illness at the time of convalescent plasma therapy, 25\% (4/16) progressed to ARDS after receiving convalescent plasma (Table 5). Three of the four required mechanical ventilation and two of the four died. One of those patients received convalescent plasma 18 days following onset of symptoms and died of refractory shock in ICU while on ventilator support. The other patient received convalescent plasma 16 days following symptom onset, developed respiratory failure 
secondary to ARDS, and was placed on comfort measures at the request of the family. The remainder $(14 / 16,88 \%)$ did not progress to ARDS, recovered with resolution of COVID-19 pneumonia, and were discharged from the hospital.

In the patients with critical illness at the time plasma therapy, 10/22 (45\%) recovered with resolution of ARDS and restoration of organ function and left the hospital. Of the $12 / 22(55 \%)$ who died, six died of refractory shock while on ventilator support with evidence of pneumoperitonium in four of them; three patients died of refractory respiratory failure with terminal extubation; two died of complications of upper airway edema; and one patient died of an acute cardiac complication.

Mean hospital length of stay was 25.6 days (95\% CI 20.8-30.4) (Table 5). Length of stay was significantly shorter in the severe illness group (15.4 days, 95\% CI 9.3-21.6) compared to patients in the critical illness group (33.0 days, 95\% CI 27.3-38.7) $(p<0.01)$. Statistical analyses showed that patients treated earlier in the course of COVID-19 disease (severe group) had significantly lower hospital mortality $(p=0.02)$ and shorter hospital length of stay $(p<0.01)$ after convalescent plasma therapy compared to patients that were treated later in their disease course in presence of ARDS (critical group) (Table 5). Other prognostic factors that were significantly associated with good clinical outcomes included shorter durations between symptoms onset and convalescent plasma administration $(p<0.01)$, and hospital admission and administration of convalescent plasma $(p<0.01)$.

\section{DISCUSSION}

Among this group of hospitalized patients with severe or critical COVID-19 who received convalescent plasma with adequate total anti-SARSCoV-2 antibody titer (1:320), only one patient experienced a transient transfusion reaction. This low rate of adverse event secondary to convalescent plasma therapy is consistent with recent published literature $[22,23]$. The overall hospital mortality among our study patients was $37 \%$. However, patients who received convalescent plasma early in the disease course (severe illness group) as compared to the patients that received convalescent plasma later in disease progression (critical illness group) had significantly lower hospital mortality 13\% vs $55 \%(p<0.02)$ and shorter mean hospital length of stay 15.4 vs 33 days $(p<0.01)$. In addition, only four patients $(25 \%)$ in the severe illness group developed ARDS, with three of them needing invasive ventilation support following convalescent plasma therapy. Two of the three recovered and were discharged.

It is important to understand the timeline and dynamics of COVID-19 hospitalizations in Connecticut and Western Massachusetts at the time when we launched our research study. Our study patients presented initially to the hospital with an average of 7.3 days (95\% CI 6.4-8.2) from symptoms' onset to hospitalization, and $97 \%(37 / 38)$ of the patients had moderate to severe disease without evidence of ARDS or urgent need for invasive ventilation support upon admission. By the time we enrolled our first patient in late April 2020, hospitals participating in the study were at their peak COVID19 census, with a large number of seriously ill patients who had been hospitalized for an average of 2 weeks, and were not improving with supportive care or medications (see Table 5). Some of those patients deteriorated and needed ICU care and ventilator support for an average of 7 days prior to enrollment. Many had severe lung damage and multi-organ failure. In the early phase of our study, physicians enrolled mostly patients in this critical illness category. In the majority of cases, patients died as a result of secondary irreversible complications of COVID-19. As our study progressed, physicians started enrolling patients earlier in their disease course and hospital stay before respiratory status deterioration. Recently published interim analysis from the Mayo Clinic Expanded Access Program for convalescent plasma in the USA reported similar experience [23].

Upon admission to the hospital, the two cohorts were similar in their demographic characteristics, pre-illness functional status, comorbidities, initial clinical presentation to 
the hospital, and initial disease severity. However, at the time of convalescent plasma administration, the groups diverged on their disease severity and duration from disease onset to plasma therapy. The patients in our study who received convalescent plasma earlier in their disease course (severe illness group) had significantly more favorable primary and secondary clinical outcomes as compared to the critical group. We speculate that convalescent plasma given earlier in the disease course, and with adequate anti-SARS-CoV-2 antibodies arrested the progression to irreversible complications like ARDS or organ failure. In addition, we found that patients who survived in both groups had shorter times between onset of symptoms and convalescent plasma administration compared to those that died. Our patients received convalescent plasma with at least 1:320 titer of total anti-SARS-CoV-2 antibody, with majority of them receiving two consecutive units of $200 \mathrm{~mL}$. This was done to maximize the potential therapeutic effect of the neutralizing anti-SARS-CoV-2 antibody. To our knowledge, there are no published reports with similar treatment strategy. The combination of using two units of convalescent plasma and high anti-SARS-CoV-2 antibody titer could have accounted for their relatively large therapeutic effect relative to other studies.

The literature suggests that convalescent plasma may be more beneficial when administered sooner to disease onset [16, 17]. Data recently published on COVID-19 suggested favorable clinical outcomes when convalescent plasma is given earlier in the course of disease [21-25, 27-29], and with higher content of antiSARS-CoV-2 antibody [27, 29]. Our finding is consistent with the literature that treating patients with COVID-19 disease with convalescent plasma earlier in the disease course, and within the first 2 weeks following symptom onset may promote recovery [30]. Perhaps earlier treatment with convalescent plasma with adequate anti-SARS-CoV-2 antibodies allows antibodies to neutralize the virus before irreversible complications [19]. Vasopressor, antibiotics, and renal replacement therapy were utilized at higher rate in the critical group-we speculate that these therapies were proxies for serious and refractory complications among critically ill patients that could not be reversed by administration of convalescent plasma. We also speculate that the difference in hydroxychloroquine utilization between the two groups is likely a reflection of the change of evidence in association with hydroxychloroquine efficacy and safety and subsequent change in practice during our study.

The published literature on mortality of hospitalized patients with COVID-19 and ARDS needing invasive ventilatory support is evolving, as earlier published reports significantly underestimated hospital mortality when significant numbers of patients included in the analyses were still in the hospital [10, 14, 31]. Recent reports from Germany, Italy, and the UK estimated the mortality rate in this population to be at least $48-55 \%$, which is consistent with our experience [31-33]. It is important to note that in our study, we only enrolled patients with severe or critical disease per the emergency IND issued by the US FDA. Many patients in the critical illness group had been hospitalized for an extended period and sustained serious lung damage prior to receiving convalescent plasma, and many remained hospitalized for weeks prior to death or discharge. Our enrolled patients may have been sicker and further along in the course of disease compared to other non-enrolled patients with COVID-19 in the ICU. Similar to the Mayo CP project, our study was pragmatic, and patients were enrolled on the basis of the judgment of their treating physicians.

As a result, our study may have been subject to "compassionate selection bias" toward sicker, and some were dying patients for whom nothing else could be done. This is an important limitation of our study and other studies that were approved under the emergency IND for convalescent plasma solicited by the FDA. Other important limitations in this study include its open-label design, no placebo control group, and modest sample size. However, despite the study design and power limitations, one interpretation of this result is that the therapeutic effect of convalescent plasma, when given early in disease course and in adequate amount, is so strong that signals of efficacy 
break through all the confounding noise to reduce mortality. Our research is important as it meaningfully contributes to the question whether convalescent plasma is safe, and it sheds light on important factors that are associated with favorable outcomes including recovery and survival.

\section{CONCLUSIONS}

For patients with severe or critical COVID-19 disease, convalescent plasma from recovered patients with COVID-19 is safe and has the potential for positive impact on clinical outcomes including recovery and survival if given early in the course of the disease, and in adequate amount. Our study makes a strong case for the importance of pursing a randomized placebo controlled trial focused on enrolling patients early in the course of their disease to further explore experimentally the efficacy and effectiveness of convalescent plasma with adequate anti-SARS-CoV-2 antibodies in COVID19.

\section{ACKNOWLEDGEMENTS}

We thank the donors, patients and their families for contributing to this work. We also thank the physicians from the Trinity Health of New England Hospitals who enrolled patients in this study at Johnson Memorial Hospital, Stafford, Connecticut (Ian Tucker); Mercy Medical Center, Springfield Massachusetts (Robert Roose, Laurie Loiacono, and Vikram Sondi); Saint Francis Hospital and Medical Center, Hartford Connecticut (Phillip Roland, Jessica AbrantesFigueiredo, Daniel Gerardi, Prashant Grover, and Gagandeep Singh), and St. Mary's Hospital in Waterbury, Connecticut (Paul Porter and Bethel Shiferaw); the New York Blood Center and Rhode Island Blood Center for assisting with the plasma collection process; Ernst J. Schaeffer and Margaret R Diffenderfer, Boston Heart Diagnostics in Massachusetts, for serology testing; Collen Lima, Jessica McKenzie, Lisa Cook, Jennifer Puff, Mary Onoroski, and Patricia Nabors for their work with donors, specimen collection and plasma delivery; Kelly Batch, Donna Sobinski, Cynthia Considine, Brian Masthay, Christina Maxwell and Robert Wilke from the Trinity Health of New England EPIC team for building the study in the electronic health record and assisting with study implementation.

Funding. This study was supported by funds from Trinity Health of New England, a not-forprofit healthcare organization. The Rapid Service Fees were funded by the authors.

Authorship. All named authors meet the International Committee of Medical Journal Editors (ICMJE) criteria for authorship for this article, take responsibility for the integrity of the work as a whole, and have given their approval for this version to be published.

Disclosures. Danyal Ibrahim, Latha Dulipsingh, Lisa Zapatka, Reginald Eadie, Rebecca Crowell, Kendra Williams, Dorothy B. Wakefield, Lisa Cook, Jennifer Puff and Syed A. Hussain declare that they have no conflicts of interests and no competing interests.

Compliance with Ethics Guidelines. The study protocol was approved by the Trinity Health of New England Institutional Review Board (\#SFH-20-23). The research study was performed in accordance with the Helsinki Declaration of 1964, and its later amendments. Informed consent was provided by either the patient or the patient's legally authorized representative (LAR).

Data Availability. The datasets generated during and/or analyzed during the current study are available from the corresponding author on reasonable request.

Open Access. This article is licensed under a Creative Commons Attribution-NonCommercial 4.0 International License, which permits any non-commercial use, sharing, adaptation, distribution and reproduction in any medium or format, as long as you give appropriate credit to the original author(s) and the source, provide a link to the Creative Commons licence, and 
indicate if changes were made. The images or other third party material in this article are included in the article's Creative Commons licence, unless indicated otherwise in a credit line to the material. If material is not included in the article's Creative Commons licence and your intended use is not permitted by statutory regulation or exceeds the permitted use, you will need to obtain permission directly from the copyright holder. To view a copy of this licence, visit http://creativecommons.org/licenses/by$\mathrm{nc} / 4.0 /$.

\section{REFERENCES}

1. Johns Hopkins University \& Medicine. COVID-19 dashboard by the Center for Systems Science and Engineering (CSSE) at Johns Hopkins University (JHU). https://coronavirus.jhu.edu/map.html. Accessed 6 Sept 2020.

2. Centers for Disease Control and Prevention. Interim clinical guidance for management of patients with confirmed coronavirus disease (COVID-19). 2020. https://www.cdc.gov/ coronavirus/2019-ncov/hcp/clinical-guidance-man agement-patients.html. Accessed 3 Aug 2020.

3. He J, Guo Y, Mao R, Zhang J. Proportion of asymptomatic coronavirus disease 2019 (COVID19): a systematic review and meta-analysis. J Med Virol. 2020. https://doi.org/10.1002/jmv.26326.

4. Park M, Cook AR, Lim JT, Sun Y, Dickens BL. A systematic review of COVID-19 epidemiology based on current evidence. J Clin Med. 2020;9(4):967.

5. Gautier JF, Ravussin Y. A new symptom of COVID19: loss of taste and smell. Obesity (Silver Spring). 2020;28(5):848.

6. Lu L, Zhang H, Zhan M, et al. Preventing mortality in COVID-19 patients: which cytokine to target in a raging storm? Front Cell Dev Biol. 2020;8:677.

7. Chen G, Wu D, Guo W, et al. Clinical and immunologic features in severe and moderate coronavirus disease 2019. J Clin Invest. 2020;130(5):2620-9.

8. Deng Y, Liu W, Liu K, et al. Clinical characteristics of fatal and recovered cases of coronavirus disease 2019 (COVID-19) in Wuhan, China: a retrospective study. Chin Med J (Engl). 2020;133(11):1261-7.
9. Liu W, Tao ZW, Wang L, et al. Analysis of factors associated with disease outcomes in hospitalized patients with 2019 novel coronavirus disease. Chin Med J (Engl). 2020;133(9):1032-8.

10. Armstrong RA, Kane AD, Cook TM. Outcomes from intensive care in patients with COVID-19: a systematic review and meta-analysis of observational studies. Anaesthesia. 2020;75(10):1340-9.

11. Quah P, Li A, Phua J. Mortality rates of patients with COVID-19 in the intensive care unit: a systematic review of the emerging literature. Crit Care. 2020;24(1):285.

12. Chen J, Qi T, Liu L, et al. Clinical progression of patients with COVID-19 in Shanghai, China. J Infect. 2020;80(5):e1-e6.

13. Bonow RO, Hernandez AF, Turakhia M. Hydroxychloroquine, coronavirus disease 2019, and QT prolongation. JAMA Cardiol. 2020.. https://doi.org/ 10.1001/jamacardio.2020.1782.

14. Gupta S, Hayek SS, Wang W, et al. Factors associated with death in critically ill patients with coronavirus disease 2019 in the US. JAMA Intern Med. 2020 . https://doi.org/10.1001/jamainternmed. 2020.3596 .

15. US Department of Health and Human Services Food and Drug Administration (FDA). Investigational COVID-19 convalescent plasma: emergency INDs. March 2020. https://www.fda.gov/vaccines-bloodbiologics/investigational-new-drug-ind-or-deviceexemption-ide-process-cber/investigational-covid19-convalescent-plasma. Accessed 10 Apr 2020.

16. Mair-Jenkins J, Saavedra-Campos M, Baillie JK, et al. The effectiveness of convalescent plasma and hyperimmune immunoglobulin for the treatment of severe acute respiratory infections of viral etiology: a systematic review and exploratory metaanalysis. J Infect Dis. 2015;211(1):80-90.

17. Sun $\mathrm{M}, \mathrm{Xu} \mathrm{Y}, \mathrm{He} \mathrm{H}$, et al. Potential effective treatment for COVID-19: systematic review and metaanalysis of the severe infectious disease with convalescent plasma therapy. Int J Infect Dis. 2020;98: 334-46.

18. Zhang L, Pang R, Xue X, et al. Anti-SARS-CoV-2 virus antibody levels in convalescent plasma of six donors who have recovered from COVID-19. Aging (Albany NY). 2020;12(8):6536-42.

19. Duan K, Liu B, Li C, et al. Effectiveness of convalescent plasma therapy in severe COVID-19 patients. Proc Natl Acad Sci USA. 2020;117(17): 9490-6. 
20. Shen C, Wang Z, Zhao F, et al. Treatment of 5 critically ill patients with COVID-19 with convalescent plasma. JAMA. 2020;323(16):1582-9.

21. Li L, Zhang W, Hu Y, et al. Effect of convalescent plasma therapy on time to clinical improvement in patients with severe and life-threatening COVID19: a randomized clinical trial. JAMA. 2020;324(5): 460-70.

22. Joyner MJ, Wright RS, Fairweather D, et al. Early safety indicators of COVID-19 convalescent plasma in 5000 patients. J Clin Invest. 2020;130(9):4791-7.

23. Joyner MJ, Bruno KA, Klassen SA, et al. Safety update: COVID-19 convalescent plasma in 20,000 hospitalized patients. Mayo Clin Proc. 2020;95(9): 1888-97.

24. Hartman WR, Hess AS, Connor JP. Hospitalized COVID-19 patients treated with convalescent plasma in a mid-size city in the midwest. Res Sq. 2020. https://doi.org/10.21203/rs.3.rs-39447/v1.

25. Salazar E, Perez KK, Ashraf M, et al. Treatment of coronavirus disease 2019 (COVID-19) patients with convalescent plasma. Am J Pathol. 2020;190(8): 1680-90.

26. US Department of Health and Human Services Food and Drug Administration (FDA). COVID-19: developing drugs and biological products for treatment or prevention guidance for industry, May 2020. https://www.fda.gov/media/137926/download. Accessed 26 July 2020.

27. Salazar E, Christensen PA, Graviss EA, et al. Treatment of COVID-19 patients with convalescent plasma reveals a signal of significantly decreased mortality. Am J Pathol. 2020:S0002-9440(20)303709.

28. Perotti C, Baldanti F, Bruno R, et al. Mortality reduction in 46 severe Covid-19 patients treated with hyperimmune plasma. A proof of concept single arm multicenter trial. Haematologica. 2020: 261784.

29. Joyner MJ, Senefeld JW, Klassen SA, et al. Effect of convalescent plasma on mortality among hospitalized patients with COVID-19: initial three-month experience. medRxiv. https://doi.org/10.1101/ 2020.08.12.20169359.

30. Rojas M, Rodríguez Y, Monsalve DM, et al. Convalescent plasma in Covid-19: possible mechanisms of action. Autoimmun Rev. 2020:102554.

31. Grasselli G, Greco M, Zanella A, et al. Risk factors associated with mortality among patients with COVID-19 in intensive care units in Lombardy, Italy. JAMA Intern Med. 2020:3539.

32. Karagiannidis C, Mostert C, Hentschker C, et al. Case characteristics, resource use, and outcomes of 10021 patients with COVID-19 admitted to 920 German hospitals: an observational study. Lancet Respir Med. 2020;S2213-2600(20):30316-7.

33. Intensive Care National Audit Research Center (ICNARC). ICNARC report on COVID-19 in critical care, July 2020. https://www.icnarc.org/ DataServices/Attachments/Download/. Accessed 6 Sep 2020. 